\title{
Large-amplitude late-time radio variability in GRB 151027B ${ }^{\star}$
}

\author{
J. Greiner ${ }^{1}$, J. Bolmer ${ }^{1,2}$, M. Wieringa ${ }^{3}$, A. J. van der Horst ${ }^{4,5}$, D. Petry ${ }^{6}$, S. Schulze ${ }^{7}$, F. Knust ${ }^{1}$, \\ G. de Bruyn ${ }^{8, \uparrow}$, T. Krühler ${ }^{1}$, P. Wiseman ${ }^{1, \star \star}$, S. Klose ${ }^{9}$, C. Delvaux ${ }^{1}$, J. F. Graham ${ }^{1}$, D. A. Kann ${ }^{9}, 10$, \\ A. Moin ${ }^{11}$, A. Nicuesa-Guelbenzu ${ }^{9}$, P. Schady ${ }^{1}$, S. Schmidl ${ }^{9}$, T. Schweyer ${ }^{1}$, M. Tanga ${ }^{1}$, S. Tingay $^{12}$, \\ H. van Eerten ${ }^{13}$, and K. Varela ${ }^{1}$
}

\footnotetext{
${ }^{1}$ Max-Planck-Institut für extraterrestrische Physik, Giessenbachstr. 1, 85748 Garching, Germany e-mail: jcg@mpe.mpg.de

${ }^{2}$ European Southern Observatory, Alonso de Cordova 3107, Vitacura, Casilla 19001, Santiago 19, Chile

${ }^{3}$ CSIRO Astronomy \& Space Science, Locked Bag 194, Narrabri, NSW 2390, Australia

${ }^{4}$ Dept. of Physics, The George Washington University, Staughton Hall, 707 22nd Street NW, Washington, DC 20052, USA

5 Astronomy, Physics and Statistics Institute of Sciences (APSIS), Staughton Hall, 707 22nd Street NW, Washington, DC 20052, USA

${ }^{6}$ ALMA Regional Centre, European Southern Observatory, Karl-Schwarzschild-Strasse 2, 85748 Garching, Germany

7 Dept. of Particle Physics and Astrophysics, Faculty of Physics, Weizmann Institute of Science, Rehovot 76100, Israel

8 ASTRON, Postbus 2, 7900 AA Dwingeloo, The Netherlands

9 Thüringer Landessternwarte Tautenburg, Sternwarte 5, 07778 Tautenburg, Germany

${ }^{10}$ Instituto de Astrofísica de Andalucía (IAA-CSIC), Glorieta de la Astronomia s/n, 18008 Granada, Spain

${ }^{11}$ Physics Department, United Arab Emirates University, P.O. Box 15551, Al-Ain, United Arab Emirates

12 Curtin Institute of Radio Astronomy, GPO Box U1987, Perth, Western Australia 6845, Australia

${ }^{13}$ Dept. of Physics, University of Bath, Claverton Down, Bath BA2 7AY, UK
}

Received 10 August 2017 / Accepted 6 February 2018

\begin{abstract}
Context. Deriving physical parameters from gamma-ray burst (GRB) afterglow observations remains a challenge, even 20 years after the discovery of afterglows. The main reason for the lack of progress is that the peak of the synchrotron emission is in the sub-mm range, thus requiring radio observations in conjunction with X-ray/optical/near-infrared data in order to measure the corresponding spectral slopes and consequently remove the ambiguity with respect to slow vs. fast cooling and the ordering of the characteristic frequencies.

Aims. We have embarked on a multifrequency, multi-epoch observing campaign to obtain sufficient data for a given GRB that allows us to test the simplest version of the fireball afterglow model.

Methods. We observed GRB 151027B, the 1000th Swift-detected GRB, with GROND in the optical-near-IR, ALMA in the submillimeter, ATCA in the radio band; we combined this with public Swift/XRT X-ray data.

Results. While some observations at crucial times only return upper limits or surprising features, the fireball model is narrowly constrained by our data set, and allows us to draw a consistent picture with a fully determined parameter set. Surprisingly, we find rapid, large-amplitude flux density variations in the radio band which are extreme not only for GRBs, but generally for any radio source. We interpret them as scintillation effects, though their extreme nature requires the scattering screen to be at a much smaller distance than usually assumed, multiple screens, or a combination of the two.

Conclusions. The data are consistent with the simplest fireball scenario for a blast wave moving into a constant-density medium, and slow-cooling electrons. All fireball parameters are constrained at or better than a factor of 2, except for the density and the fraction of the energy in the magnetic field which has a factor of 10 uncertainty in both directions.
\end{abstract}

Key words. gamma-ray burst: general - gamma-ray burst: individual: GRB 151027B - radiation mechanisms: non-thermal radio continuum: ISM - techniques: photometric

\section{Introduction}

It is widely accepted that long-duration gamma-ray bursts (GRBs) are related to the death of massive stars (Hjorth et al. 2003; Stanek et al. 2003). Due to their large $\gamma$-ray luminosity, they can be detected to very high redshift, and thus can provide a unique probe into the early Universe. How the afterglow

\footnotetext{
* This paper makes use of the following data: ATCA: Proposal C2955 (PI: Greiner), ALMA: ADS/JAO.ALMA\#2015.1.01558.T (PI: Schulze).

$\star \star$ Present address: School of Physics and Astronomy, Univ. of Southampton, Southampton S017 1BJ, UK.

${ }^{\dagger}$ Deceased.
}

emission evolves both in frequency space and with time depends on the properties of the burst environment (e.g., gas density profile, dust) and on the progenitor itself (e.g., temporal energy injection profile, as well as mass, rotation, and binarity, all of which influence the density and structure of the circumburst medium, e.g., Yoon et al. 2012).

When the relativistically expanding blast wave interacts with the circumburst medium, an external shock is formed, the macroscopic properties of which are well understood. Under the implicit assumptions that the electrons undergo Fermi acceleration at the relativistic shock to a power-law distribution, their dynamics can be expressed in terms of four main parameters: 
(1) the total internal energy in the shocked region as released in the explosion, (2) the electron density $n$ and radial profile of the surrounding medium, (3) the fraction of the shock energy that goes into electrons $\epsilon_{e}$, and (4) the ratio of the magnetic field energy density to the total energy $\epsilon_{B}$. Measuring the energetics or the energy partition $\left(\epsilon_{e} / \epsilon_{B}\right)$ has been challenging, and observations at multiple passbands have thus far only been possible for a dozen of the more than 1000 GRB afterglows detected so far.

The observational difficulty of establishing whether the observed synchrotron spectrum is in the fast or slow cooling stage introduces a degeneracy when attempting to explain the spectrum in terms of the physical model parameters. The minimal and simplest afterglow model has five parameters (not counting the distance/redshift). The degeneracy between many of these parameters makes it even more difficult to draw firm conclusions. Thus, it is not surprising that many previous attempts had to compromise whenever assumptions were made about individual parameters (e.g., Panaitescu \& Kumar 2002; Yost et al. 2003; Chandra et al. 2008; Cenko et al. 2010; Greiner et al. 2013; Laskar et al. 2014; Varela et al. 2016), but contradictions between analyses with different assumptions surfaced only in the rare cases where the same GRB afterglows were analyzed based on different data sets (e.g., McBreen et al. 2010; Cenko et al. 2011).

Here, we report our multi-epoch, multifrequency observations of GRB 151027B, in an attempt to collect an exhaustive data set that would allow us to determine all these parameters.

\section{GRB 151027B detection and afterglow observations}

\subsection{GRB prompt and afterglow detection}

GRB 151027B was detected by the Swift (Gehrels et al. 2004) Burst Alert Telescope (BAT, Barthelmy et al. 2005) on 2015 October 27 at $T_{0}=22: 40: 40$ UT (MJD $\left.=57322.944907\right)$ as the 1000th Swift burst (Ukwatta et al. 2015). The prompt light curve shows a complex structure with several overlapping peaks that starts at $\sim T_{0}$ and extends for about $100 \mathrm{~s}$, leading to a formal

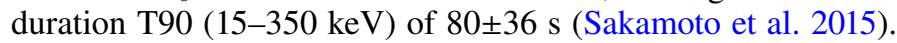
Swift slewed immediately to the BAT-derived position, allowing the X-ray afterglow to be discovered readily with the Swift X-ray telescope (XRT, Burrows et al. 2005) with a 4 " accurate position (later refined to 1". 8 ). This in turn allowed the discovery of the optical afterglow one hour later by the Nordic Optical Telescope (Malesani et al. 2015), and a redshift determination of $z=4.063$ with VLT/X-shooter four hours after that (Xu et al. 2015). In addition to our GROND observations (see below), detections of the optical afterglow were also reported by MASTER (Buckley et al. 2015), RATIR (Watson et al. 2015) and the $2 \mathrm{~m}$ Faulkes Telescope North in Hawaii (Dichiara et al. 2015). Swift/UVOT did not detect the afterglow, consistent with the redshift and galactic foreground extinction (Breeveld et al. 2015).

\subsection{GROND observations}

Observations with GROND (Greiner et al. 2008) started on 2015 October 28 at 06:26 UT, about $8 \mathrm{hr}$ after the trigger, at a Moon distance of only $37^{\circ}$. Simultaneous imaging in $g^{\prime} r^{\prime} i^{\prime} z^{\prime} J H K_{\mathrm{s}}$ continued for several further epochs (see the observation log in Table 1) until 2015 November 18, when the afterglow could no longer be detected. During the night of November 5-6, a field with SDSS coverage $(\operatorname{RA}(2000.0)=03 \mathrm{~h} 45 \mathrm{~m}$,

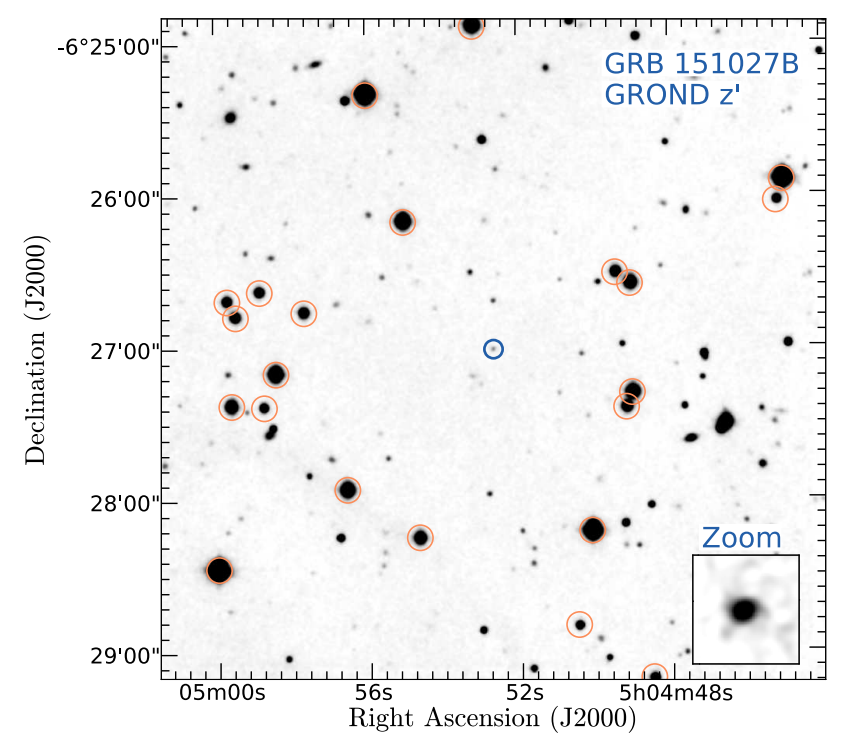

Fig. 1. Finding chart of the afterglow of GRB 151027B based on a GROND $z^{\prime}$-band image, with secondary standard stars of Table A.1 encircled. North is up and east to the left.

$\left.\operatorname{Dec}(2000.0)=-06^{\circ} 15^{\prime}\right)$ was observed immediately after the GRB field under photometric conditions.

GROND data have been reduced in the standard manner (Krühler et al. 2008) using pyraf/IRAF (Tody 1993; Küpcü Yoldaş et al. 2008). The optical-near-IR (NIR) imaging was calibrated against the primary $\operatorname{SDSS}^{1}$ standard star network, or catalogued magnitudes of field stars from the SDSS in the case of $g^{\prime} r^{\prime} i^{\prime} z^{\prime}$ observations, or the 2MASS catalog for $J H K_{\mathrm{s}}$ imaging. This results in typical absolute accuracies of \pm 0.03 mag in $g^{\prime} r^{\prime} i^{\prime} z^{\prime}$ and $\pm 0.05 \mathrm{mag}$ in $J H K_{\mathrm{s}}$. Comparison stars covered by the finding chart of GRB 151027B (Fig. 1) are given in Table A.1.

Despite its high redshift, the afterglow was detected in all seven bands (Table 1) at a common position of RA(2000.0), $\operatorname{Dec}(2000.0)=76.21955,-6.45029$, or 05:04:52.69-06:27:01.1, with a $1 \sigma$ error of \pm 0.25 . This is fully consistent with both the UVOT-corrected Swift/XRT position (Osborne et al. 2015) and the NOT-derived position (Malesani et al. 2015).

\subsection{ATCA observations}

We observed the field of GRB 151027B under program C2955 (PI: Greiner) simultaneously at 5.5 and $9 \mathrm{GHz}$ with the Australia Telescope Compact Array (ATCA), beginning at October $30.54 \mathrm{UT}$ for $3.3 \mathrm{hr}$; the corresponding detection at $9 \mathrm{GHz}$ was reported earlier (Greiner et al. 2015b). Over the following three months, we observed the GRB 151027B position at another seven epochs. A summary of the observing log, including the telescope configuration, is given in Table 2. The observations were mostly performed with the CFB $1 \mathrm{M}-0.5 \mathrm{~K}$ mode, providing 2048 channels per $2048 \mathrm{MHz}$ continuum intermediate frequency (IF; $1 \mathrm{MHz}$ resolution) and 2048 channels per $1 \mathrm{MHz}$ zoom band $(0.5 \mathrm{kHz}$ resolution). Data analysis was done using the standard software package MIRIAD (Sault et al. 1995), applying appropriate bandpass, phase, and flux calibrations. The quasar 0458-020 was used as phase and 1934-638 as flux calibrator. Multifrequency synthesis images were constructed using robust weighting (robust $=0$ ) and the full bandwidth between its flagged edges. The noise was determined by estimating the root mean square (rms) in emission-free parts of the cleaned map.

\footnotetext{
1 http://www.sdss.org
} 
Table 1. GROND observations; all in the $\mathrm{AB}$ system, not corrected for Galactic foreground extinction corresponding to $E(B-V)=0.18$ mag $\left(A_{V}=0.55 \mathrm{mag}\right)$ (Schlafly \& Finkbeiner 2011).

\begin{tabular}{lccccccc}
\hline \hline $\begin{array}{l}\text { Time after } T_{0} \\
(\mathrm{~s})\end{array}$ & $\begin{array}{c}g^{\prime} \\
(\mathrm{mag})\end{array}$ & $\begin{array}{c}r^{\prime} \\
(\mathrm{mag})\end{array}$ & $\begin{array}{c}i^{\prime} \\
(\mathrm{mag})\end{array}$ & $\begin{array}{c}z^{\prime} \\
(\mathrm{mag})\end{array}$ & $\begin{array}{c}J \\
(\mathrm{mag})\end{array}$ & $\begin{array}{c}H \\
(\mathrm{mag})\end{array}$ & $\begin{array}{c}K_{\mathrm{s}} \\
(\mathrm{mag})\end{array}$ \\
\hline $28830 \pm 903$ & $22.16 \pm 0.23$ & $20.41 \pm 0.05$ & $19.78 \pm 0.04$ & $19.58 \pm 0.04$ & $19.47 \pm 0.09$ & $18.96 \pm 0.08$ & $19.09 \pm 0.16$ \\
$30948 \pm 1125$ & $22.17 \pm 0.26$ & $20.59 \pm 0.07$ & $20.02 \pm 0.06$ & $19.66 \pm 0.05$ & $19.48 \pm 0.10$ & $19.19 \pm 0.10$ & $19.10 \pm 0.20$ \\
$33291 \pm 1129$ & $22.69 \pm 0.24$ & $20.62 \pm 0.04$ & $20.00 \pm 0.04$ & $19.67 \pm 0.04$ & $19.52 \pm 0.07$ & $19.17 \pm 0.08$ & $19.10 \pm 0.14$ \\
$35634 \pm 1130$ & $22.45 \pm 0.16$ & $20.64 \pm 0.04$ & $20.08 \pm 0.04$ & $19.77 \pm 0.04$ & $19.54 \pm 0.07$ & $19.39 \pm 0.08$ & $19.24 \pm 0.14$ \\
$112431 \pm 6317$ & $>23.6$ & $22.05 \pm 0.07$ & $21.53 \pm 0.07$ & $21.18 \pm 0.06$ & $21.10 \pm 0.15$ & $20.73 \pm 0.16$ & $20.36 \pm 0.24$ \\
$202273 \pm 1606$ & $>24.0$ & $22.57 \pm 0.09$ & $22.09 \pm 0.09$ & $21.85 \pm 0.08$ & $21.80 \pm 0.25$ & $21.53 \pm 0.29$ & $>20.9$ \\
$804300 \pm 6436$ & $>25.8$ & $24.75 \pm 0.16$ & $24.33 \pm 0.21$ & $23.87 \pm 0.23$ & $>22.3$ & $>21.9$ & $>21.5$ \\
$1838665 \pm 3570$ & $>25.5$ & $>25.7$ & $>24.9$ & $>24.7$ & $>22.4$ & $>22.0$ & $>21.4$ \\
\hline
\end{tabular}

Table 2. ATCA observing details.

\begin{tabular}{|c|c|c|c|c|c|}
\hline Date \& Start-Time & $\begin{array}{c}\text { On source } \\
\text { exposure (hr) }\end{array}$ & $\begin{array}{l}\text { Time after GRB } \\
(\text { day })^{a}\end{array}$ & $\begin{array}{c}\text { Telescope } \\
\text { configuration }\end{array}$ & $\begin{array}{c}5.5 \mathrm{GHz} \text { flux } \\
\mu \mathrm{Jy}^{b}\end{array}$ & $\begin{array}{c}9 \mathrm{GHz} \text { flux } \\
\mu \mathrm{Jy}^{b}\end{array}$ \\
\hline 2015-10-30 12:56 & 3.3 & $2.76 \pm 0.16$ & $6 \mathrm{~A}$ & $<18$ & $67 \pm 10$ \\
\hline 2015-11-02 12:00 & 3.2 & $5.74 \pm 0.19$ & $6 \mathrm{~A}$ & $73 \pm 10$ & $98 \pm 11$ \\
\hline 2015-11-11 15:36 & 5.8 & $14.70 \pm 0.19$ & $6 \mathrm{~A}$ & $76 \pm 7$ & $<15$ \\
\hline 2015-11-14 11:31 & 3.3 & $17.69 \pm 0.16$ & $6 \mathrm{~A}$ & $<26.0$ & $100 \pm 10$ \\
\hline 2015-11-16 12:00 & 6.7 & $19.72 \pm 0.17$ & $1.5 \mathrm{~A}$ & $<13.4$ & $<15.4$ \\
\hline 2015-12-02 09:36 & 8.4 & $35.67 \pm 0.21$ & $1.5 \mathrm{~A}$ & $60 \pm 11$ & $36 \pm 11$ \\
\hline 2015-12-11 10:05 & 2.5 & $44.54 \pm 0.06$ & $750 \mathrm{C}$ & $71 \pm 12$ & $<22$ \\
\hline 2016-01-22 06:30 & 5.7 & $86.48 \pm 0.16$ & EW352 & $<24$ & $<28$ \\
\hline
\end{tabular}

Notes. ${ }^{(a)}$ The error denotes the time span over which the exposure was spread to cover the $u-v$ plane. ${ }^{(b)}$ Upper limits are given at the $2 \sigma$ level.

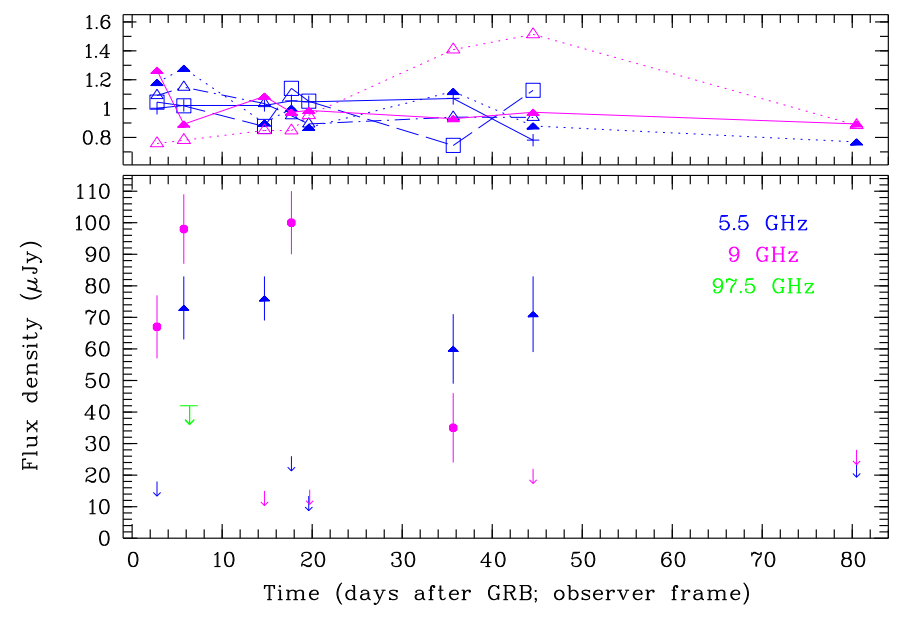

Fig. 2. Radio light curve of the afterglow of GRB 151027B at 5.5 and $9 \mathrm{GHz}$, with the ALMA $97.5 \mathrm{GHz} 2 \sigma$ upper limit overplotted (lower panel). The upper panel shows the measured fluxes of selected brighter $(130-500 \mu \mathrm{Jy})$ sources. While their nature or intrinsic variability is not known, their $<20 \%$ flux variation demonstrates that the strong fluctuations seen for the GRB 151027B afterglow (which would correspond to an amplitude between 0.2 and 2 in this graph) is not an instrumental artifact.

Given the largely varying flux levels between different observations and the large flux differences between the two frequencies, we note that the signal-to-noise ratio in most detections is so high that it is unlikely our measurements are wrong. We employed two further tests. First, for the November 14 observation, we made separate images for the top and bottom of the $9 \mathrm{GHz}$ band, resulting in flux measurements of $96 \mu \mathrm{Jy}$
(8-9 GHz) and $106 \mu \mathrm{Jy}(9-10 \mathrm{GHz})$, thus providing an internally consistent result. Second, we checked for other sources in the field for evidence of this variation, but did not find any (see Fig. 2).

\subsection{ALMA observations}

ALMA observations were triggered under proposal-ID 2015.1.01558.T (PI: S. Schulze). A band 7 (343.495 GHz) observation was performed starting on 2015 November 2 at 05:22 UT under a precipitable water vapor (PWV) of $0.71 \mathrm{~mm}$, and a band $3(97.495 \mathrm{GHz})$ observation started on 2015 November 4 at 07:45 UT under a PWV of $0.31 \mathrm{~mm}$. The data analysis was performed using the standard ALMA data analysis package CASA (McMullin et al. 2007; Petry et al. 2012), following the default calibration path also used in ALMA Quality Assurance. The final images are shown in Fig. 3.

Within the GROND error circle of 0.25 in band 3, we find a peak with a flux of $0.0619 \mathrm{mJy}$; given the rms noise of $0.0210 \mathrm{mJy}$, this corresponds to nearly $3 \sigma$. However, the area of the error circle contains 1914 spatial resolution pixels, so we expect 1914 pixels $\times 0.0016=3$ pixels to be above a $3 \sigma$ flux level. Thus, the presence of the source-like point in the error circle is compatible with a random occurrence, likely thermal noise. In fact, there are similar peaks outside the error circle.

In band 7, we find a peak within the error circle of $0.177 \mathrm{mJy}$, which (given the rms noise of $0.0496 \mathrm{mJy}$ ) corresponds to $3.5 \sigma$. However, the area of the error circle contains 7793 spatial resolution pixels, so we expect 7793 pixels $\times 0.0002=1.6$ pixels to be above a $3.5 \sigma$ flux level.

Summarizing, no source is detected in either observation, with $2 \sigma$ upper limits of $42 \mu \mathrm{Jy}$ in band $3(97.495 \mathrm{GHz}$, integrated 

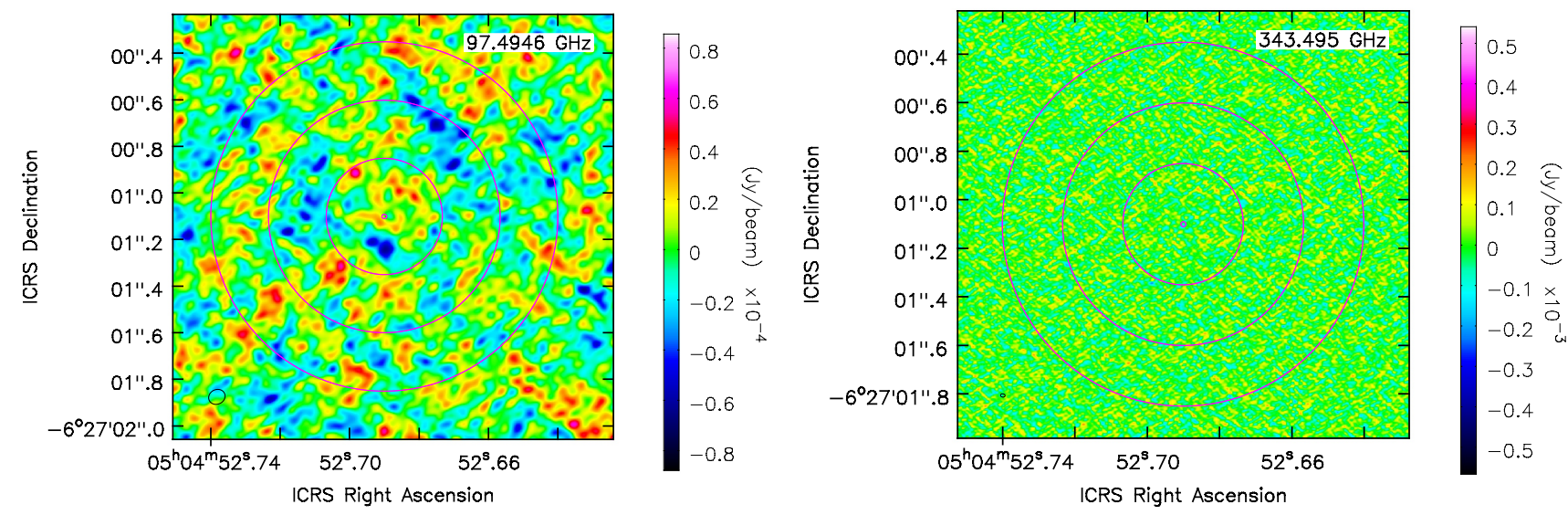

Fig. 3. ALMA images of the GRB 151027B location at band $3(97.5 \mathrm{GHz}$; left $)$ and band $7(343.5 \mathrm{GHz}$; right $)$ in the International Celestial Reference System (ICRS). The concentric circles around our best-fit GROND position are the $1 \sigma, 2 \sigma$, and $3 \sigma$ error circles. The contour in the lower left of each figure gives the size of the synthesized beam of the observation. The smaller beam size makes the band 7 image look smoother than that of band 3; the rms noise is actually worse (see text).

over a bandwidth of $7 \mathrm{GHz}$, and taking into account that only about $87 \%$ of each of the four spectral windows was used; edge channels are not good) at 7.378 days after the GRB, and $100 \mu \mathrm{Jy}$ in band $7(343.495 \mathrm{GHz}$, also with a bandwidth of $7 \mathrm{GHz})$ at 5.279 days after the GRB. These values include the primary beam correction, which is $>0.99$ because it is close to the center of the field of view.

\section{Results}

Here we analyze our data in the context of the GRB fireball model (Meszaros \& Rees 1997; Granot \& Sari 2002). Throughout this paper, we use the definition $F_{v} \propto t^{-\alpha} v^{-\beta}$, where $\alpha$ is the temporal decay index and $\beta$ is the spectral slope.

\subsection{Radio scintillation}

The large-amplitude radio variability observed in this GRB is very unusual. In the context of the canonical fireball scenario a smoothly varying afterglow would be expected, perhaps with a rapid rise and decay due to reverse shock emission, none of which is akin to our data. Moreover, we observe large variations between the simultaneously covered 5.5 and $9 \mathrm{GHz}$ bands, i.e., the inferred spectral slope changes between $<-2.3$ and $>2.9$ within days, while temporal slopes in the range $<-15$ and $>9$ over 2-3 days are implied. We are not aware of any physical process(es) in GRB jets or shocks capable of producing emission with such properties, and thus consider the afterglow radio emission to be strongly influenced by scintillation.

Interstellar scintillation effects have been observed in GRB radio light curves, and have been used to obtain indirect measures of the source size (for a recent review, see Granot \& van der Horst 2014). This method relies on the fact that propagation effects in the interstellar medium cause modulations of the flux of a compact source, while a source larger than a certain angular size will not vary (Rickett 1990). In the case of GRBs, the source is the evolving shock front of the jet, which is very compact at first but expands over time. This can result in strong modulations at early times, which get quenched at later times (Frail et al. 1997; Goodman 1997; Frail et al. 2000). These variations can be found between observations on different days, but intraday variability has also been observed in GRBs (e.g., Chandra et al. 2008; van der Horst et al. 2014). The typical procedure for relating the source size to the scintillation effects is to estimate the scintillation strength and timescale using the methods of Walker (1998) combined with the NE2001 model of the free electrons in our galaxy (Cordes \& Lazio 2002). In the strong scattering regime, there are two possible types of scintillation, refractive and diffractive. In both cases the modulation strength depends on the source size compared to the angular scale for scintillation, which ranges from a few to a few tens of microarcseconds. Diffractive scintillation gives stronger flux modulations than refractive scintillation, but the angular scale for diffractive scintillation is smaller than that for refractive scintillation. Furthermore, the former is a narrowband phenomenon while the latter is broadband, but they could both be at play in GRB afterglow observations.

The redshift of GRB 151027B is 4.063, which means that 1 arcsecond on the sky corresponds to a distance of $7.05 \mathrm{kpc}$, so 1 microarcsecond corresponds to $2.2 \times 10^{16} \mathrm{~cm}$. A size of $10^{16}-10^{17} \mathrm{~cm}$ is typical for the jet size, so strong scintillation effects are expected for this GRB, also because the high redshift of the GRB means that 40 days in the observer frame corresponds to 8 days in the source rest frame. The scintillation timescale of several hours to days that we observe for GRB 151027B is plausible, but the observed modulation seems to be too large to be accommodated within this framework. The maximum modulation index for diffractive scintillation is 1, i.e., the flux can increase or decrease by a factor of 2 due to scintillation, and the modulation index for refractive scintillation is always smaller than 1 . Both of these changes are significantly smaller than the jumps in flux that we have observed for GRB 151027B, which are more than a factor of 5 between some observations (at the $2 \sigma$ level). For instance, at $9 \mathrm{GHz}$ the flux changes from $<15 \mu \mathrm{Jy}$ at 14.7 days, to $100 \pm 10 \mu \mathrm{Jy}$ at 17.7 days, and then to $<15 \mu \mathrm{Jy}$ at 19.7 days; flux changes of more than a factor of 5, both up and down.

Given that these strong flux modulations cannot be explained by physical processes in the source itself, scintillation does seem to be the most natural way to explain the observations, as has been done for other GRBs with radio flux modulations. However, in this particular case, we have to deviate from the typical methodology applied in the modeling of scintillation effects on GRB radio light curves, due to the very large and fast modulations. One of the underlying assumptions of the usual methodology is that the scattering happens at one location, the scattering screen, which resides at a typical distance (usually $1 \mathrm{kpc}$ from the observer). However, many studies of interstellar scintillation 


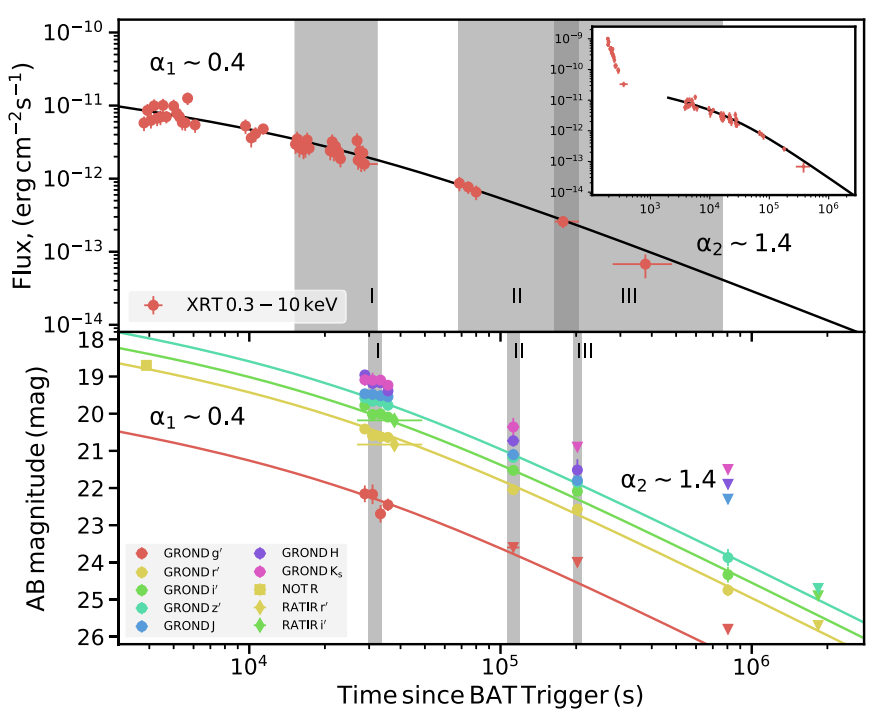

Fig. 4. Light curve of the afterglow of GRB 151027B at X-rays as observed with Swift/XRT (top), and in the optical-NIR as observed with GROND (no extinction-correction applied), complemented with two measurements by NOT and RATIR (Malesani et al. 2015; Watson et al. 2015). Error bars are plotted, but are mostly smaller than the symbol size. The vertical gray bands mark the time intervals for which the spectral energy distributions have been established (see text for more details, and Fig. 5).

with pulsars and active galactic nuclei have shown that the distance of the scattering screen is quite uncertain. Varying this distance can have strong effects on both the modulation strength and timescale. For example, some quasars have shown extreme intraday variability, indicating that their scattering screen is significantly closer than is usually assumed (Dennett-Thorpe \& de Bruyn 2002; Bignall et al. 2006; Macquart \& de Bruyn 2007; de Bruyn \& Macquart 2015). Furthermore, extragalactic sources may be shining through multiple scattering screens inside our galaxy, complicating the scintillation behavior even further. Every scattering screen will impose its own modulation strength and timescale, possibly leading to enhanced and complex scintillation behavior.

The bottom line is that the observed fluctuations in GRB 151027B can be explained by scintillation, but the large modulation amplitude and rapid variations suggests that the scattering screen is at a smaller distance, that there are multiple screens, or a combination of the two. Many more detailed studies of various radio sources, including GRB afterglows, are needed to fully probe the scintillation behavior of the interstellar medium in our local environment.

\subsection{Constraints on the fireball model}

Both the X-ray and the optical light curves can be modeled with a smoothly broken power law (Fig. 4) with $\alpha_{1}=0.44 \pm 0.19, \alpha_{2}=$ $1.44 \pm 0.14$, and $t_{b} \sim 22.5 \mathrm{ks}$, consistent with the magnitudes observed by NOT and RATIR (Malesani et al. 2015; Watson et al. 2015). These temporal slopes were used to rescale an XRT spectrum from data taken between $T_{0}+15 \mathrm{ks}$ and $T_{0}+32 \mathrm{ks}$ to the stacked GROND data taken between $T_{0}+32 \mathrm{ks}$ and $T_{0}+34$ ks (shaded gray intervals in Fig. 4). The resulting broadband spectral energy distribution (SED) is best fit with a single power law of slope $\beta=0.81 \pm 0.01$, with a negligible amount of dust $\left(A_{V}=0.01 \pm 0.01 \mathrm{mag}\right)$, independent of the extinction model (see Bolmer et al. 2018 for more details on the extinction determination, where a broken power-law model has been

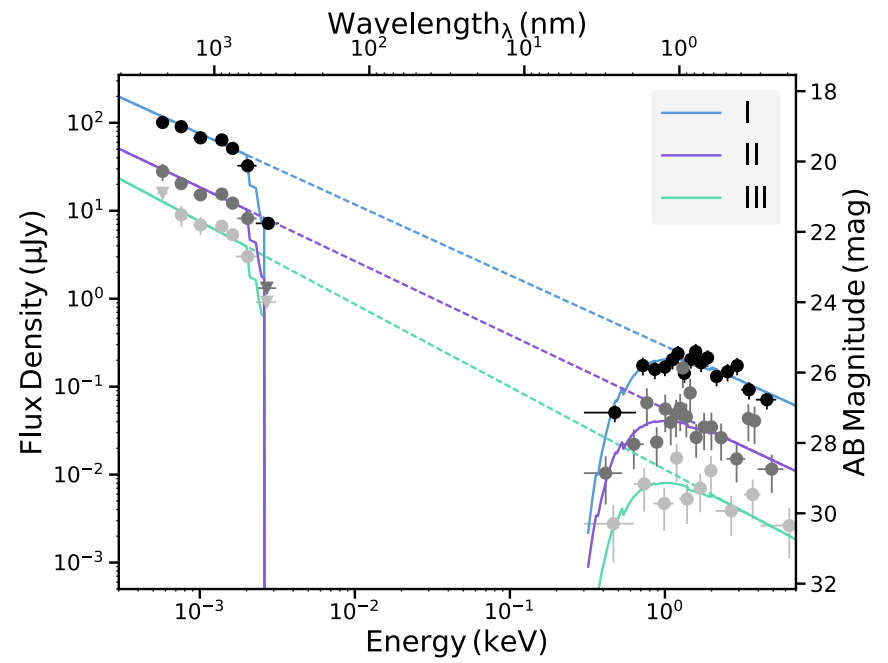

Fig. 5. Observer-frame optical-NIR to X-ray spectral energy distribution of the afterglow of GRB 151027B at the three epochs marked in Fig. 4 with the gray shading. Error bars are plotted, but are mostly smaller than the symbol size.

preferred in order to derive a conservative extinction value). In these fits, the $g^{\prime} r^{\prime}$ filters were ignored owing to additional uncertainty from absorption from the Ly $\alpha$ forest. The spectral slope in the X-ray to optical-NIR does not change with time within errors $\left(\beta_{1}=0.81 \pm 0.01, \beta_{2}=0.83 \pm 0.03\right.$, and $\left.\beta_{3}=0.89 \pm 0.07\right)$ as evidenced by the other broadband SEDs at later times (see Fig. 5), nor do the data require a spectral break at later times. The above post-break parameters are fully consistent (within $2 \sigma$ ) with an afterglow with an electron power-law distribution with $p=2.62 \pm 0.02$, evolving via slow cooling into an ISM environment where the cooling break is above the Swift/XRT upper energy boundary: measured $\alpha_{2}=1.44 \pm 0.14$ versus predicted $\alpha_{2}=1.22 \pm 0.02$. A cooling break below the GROND bands would imply $p=1.62 \pm 0.02$ and $\alpha_{2}=0.72$, inconsistent with our observed light curve. In the preferred scenario, the cooling break $v_{c}$ would move to lower frequencies proportional to $t^{-1 / 2}$. Since we also do not see any signature of a spectral break in the X-ray band up to $2 \times 10^{5} \mathrm{~s}$ after the GRB, after backextrapolation this implies that $v_{c}(31 \mathrm{ks})>20 \mathrm{keV}$. We finally note that the pre-break phase is consistent with the plateaus seen in many Swift-detected GRBs (e.g., Dainotti et al. 2017, and references therein), with the optical data (primarily NOT) fitting the picture, and with being in the same synchrotron spectral regime.

The remaining question then is the relative ordering of the peak frequency $v_{m}$ and the self-absorption frequency $v_{s a}$. Given the multiple radio detections with ATCA implies that the self-absorption frequency should be below $5 \mathrm{GHz}$ already at 2.8 days after the GRB in order for the scintillation amplitude not to exceed a factor of 10. The ALMA limits then require $v_{m}$ to be above the self-absorption frequency. Considering the canonical decrease in $v_{m}$ according to $t^{-3 / 2}$, our following two observational constraints fix the value of $v_{m}(t)$ to better than 20\%: (i) at the time of the first GROND observation, $v_{m}(31 \mathrm{ks})<1.3 \times 10^{14} \mathrm{~Hz}$, and (ii) the ALMA band 7 limit together with the interpolated optical-NIR fluxes at this epoch imply $v_{m}(5.279 \mathrm{~d})>1.8 \times 10^{12} \mathrm{~Hz}$. Back-extrapolating the latter limit to the first GROND observation (by a factor of $\left.(31 \mathrm{ks} / 456.1 \mathrm{ks})^{-3 / 2}=56.4\right)$ implies an inferred $v_{m}(31 \mathrm{ks})=$ $(1.15 \pm 0.15) \times 10^{14} \mathrm{~Hz}$.

With these observational constraints it is possible to determine the fireball parameters. We observe the following set of 

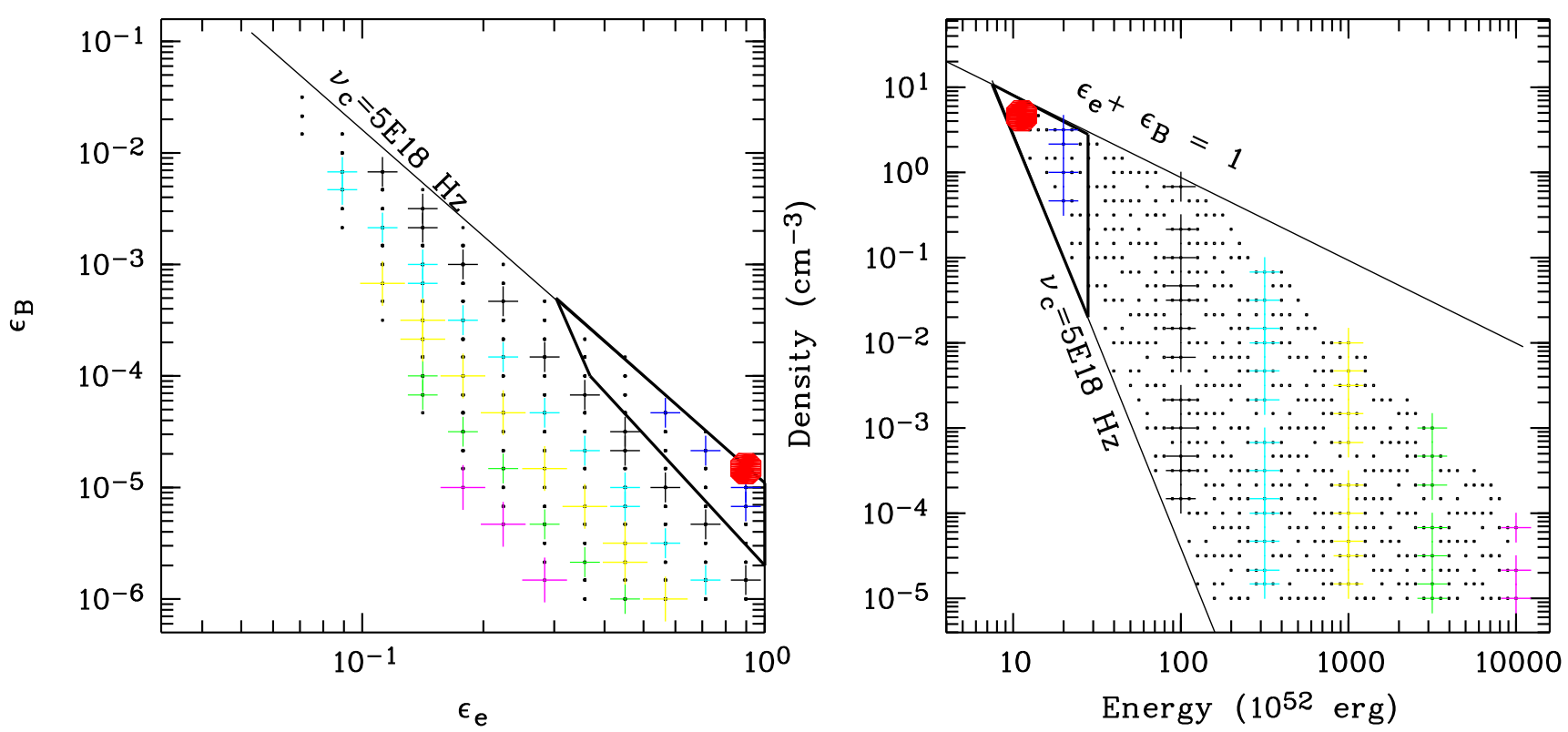

Fig. 6. Constraints on the fireball parameters for the afterglow of GRB 151027B. Small black dots delineate the allowed phase space by the four constraints at $31 \mathrm{ks}$, and colored crosses indicate different possible solutions for seven different values of the kinetic energy. The solution with the lowest kinetic energy is marked with the red-filled octagon: $E_{\text {kin }}=11.2 \times 10^{52} \mathrm{erg}$, external density $n=5 \mathrm{~cm}^{-3}, \epsilon_{e}=0.9$, and $\epsilon_{B}=1.5 \times 10^{-5}$. The thick-lined triangles enclose the allowed parameter range if $E_{\mathrm{kin}}<5 \times E_{\gamma \text {,iso }}$.

relations, all at $31 \mathrm{ks}$ after the GRB:

$v_{m}=(1.15 \pm 0.15) \times 10^{14} \mathrm{~Hz}$

$F_{v}\left(v_{m}\right)=(100 \pm 10) \mu \mathrm{Jy}$

$v_{c}>4.8 \times 10^{18} \mathrm{~Hz}(=20 \mathrm{keV})$

$F_{v}\left(v_{c}\right)<0.07 \mu \mathrm{Jy}$.

Within the canonical fireball scenario (Granot \& Sari 2002) in the slow cooling case with the ordering $v_{s a}<v_{m}<v_{c}$ and ISM density profile, the self-absorption frequency remains constant. Because it is always below $100 \mathrm{MHz}$, i.e., below our observed frequencies, for the entire allowed parameter range (see below), it does not provide any additional constraints.

These constraints on the observed frequencies and fluxes lead to bounds on the fireball parameters (see Fig. 6). While the observations do not uniquely constrain all parameters, we can use an efficiency argument to derive a likely parameter range. In the standard picture, a fraction $\epsilon_{\gamma}$ of the explosion energy is radiated in the prompt radiation (observable as $E_{\gamma, \text { iso }}$ ), and the remaining fraction ending up as kinetic energy $E_{\text {kin }}$ of the swept up ambient gas. Early observations suggested near equipartition between these two channels, though later considerations including proper error estimates suggest $\epsilon_{\gamma}$ in the range of $\sim 0.1-0.5$ (Granot et al. 2006). Assuming $\epsilon_{\gamma}=0.2$ and using $E_{\gamma, \text { iso }}(15-10000 \mathrm{keV})=(5 \pm 1) \times 10^{52}$ erg (based on a bestfit cutoff power-law of the Swift/BAT data ${ }^{2}$ giving an energy fluence of $\left.(14.7 \pm 2.6) \times 10^{-7} \mathrm{erg} \mathrm{cm}^{-2}\right)$, the GRB 151027B fireball parameters are constrained as follows: external density $n=0.03-5 \mathrm{~cm}^{-3}, \epsilon_{e}=0.3-1.0$, and $\epsilon_{B}=4 \times 10^{-4}-2 \times 10^{-6}$ (see Fig. 6). We note that our derived $\epsilon_{e}$ is higher than the majority of published afterglows, though still in the allowed range.

For the solution with the lowest kinetic energy, $E_{\text {kin }}=11.2 \times 10^{52} \mathrm{erg}$ (remaining parameters see caption

2 Provided at http://gcn.gsfc.nasa.gov/notices_s/661869/ $\mathrm{BA} /$

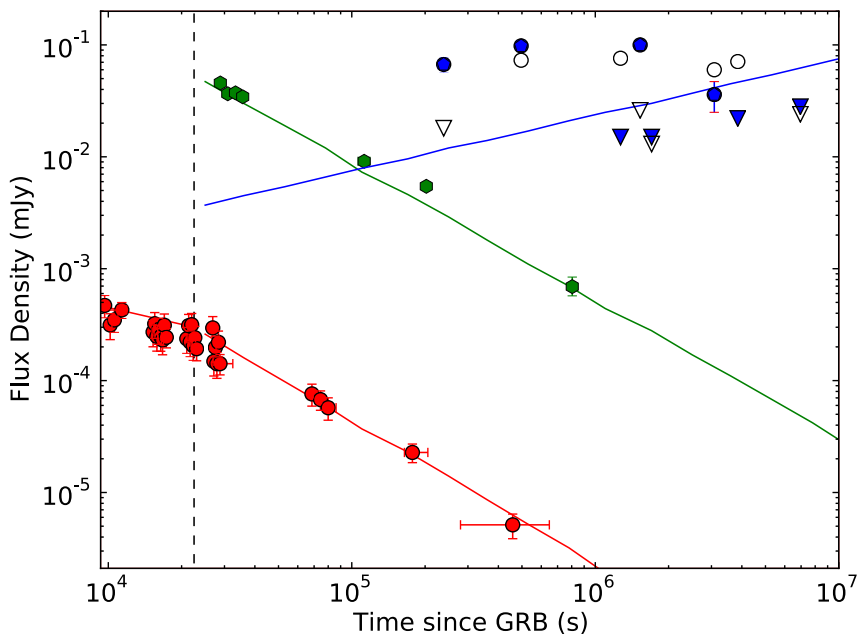

Fig. 7. Model light curves for the afterglow of GRB 151027B at X-rays (red), optical (green, extinction-corrected), and radio (blue; open and filled symbols are 5.5 and $9 \mathrm{GHz}$, respectively; circles $=$ detection, triangles $=$ upper limits) for the lowest- $E_{\text {kin }}$ parameter set. Data are drawn with error bars, which are mostly smaller than the symbol size. The vertical line denotes the break time of $22.5 \mathrm{ks}$ (see Sect. 3.2). Radio data have not been used in deriving the model, so the blue curve is actually a "predicted" light curve.

of Fig. 6), we then compute the X-ray, optical I-band, and 7 $\mathrm{GHz}$ radio (average of 5.5 and $9 \mathrm{GHz}$ ) light curves, which are shown in Fig. 7. We note that the model was derived without using constraints from the radio bands, so it is interesting that the "predicted" radio light curve in Fig. 7 corresponds roughly to the mean of the radio detections and upper limits. This implies that the scintillation interpretation of the measured radio fluxes is reasonable and that the model is reasonably good. Thus, the observed scintillation corresponds to about a factor of \pm 3 variation in either direction, but it is not a one-way excursion. 


\section{Conclusions}

Our data set of the GRB 151027B afterglow can be explained with the simplest version of the standard fireball scenario, displaying a single synchrotron spectrum evolving according to standard dynamics.

We find that the blast wave moves into a constant-density environment, in the slow cooling regime. While we do not see the characteristic movement of the cooling frequency in or through the X-ray band, the constancy of the peak flux over the full observing epoch as evidenced by our data requires a constant density profile. The derived fireball parameters are all within the range expected and discussed in the literature. For the smallest allowed kinetic energy, $\epsilon_{e}$ is pushed towards the upper limit of 1 .

After GRBs 000131, 050904, 090423, 111008A, 120521C, 130606A, 140304A, 140311A, 140515A the afterglow of GRB $151027 \mathrm{~B}$ is the tenth above a redshift of 4 that has been detected in the radio band (see the online summary table). ${ }^{3}$ Its peak spectral radio luminosity $\left(2 \times 10^{31} \mathrm{erg} / \mathrm{s} / \mathrm{Hz}\right)$ is among the top one-quarter of radio afterglows (Chandra \& Frail 2012), but certainly not exceptional. However, the large-amplitude and rapid flux fluctuations up to $9 \mathrm{GHz}$ are exceptional, and imply that scintillation plays a major role, even at 45 days ( 9 days rest-frame) post-burst.

We finally mention that the ALMA flux limits are close to the prediction of our model, so we cannot completely rule out that the $3.5 \sigma$ blob in the band 7 image is not actually the afterglow.

With the above caveats it is worth noting that this is one of the few afterglows of long-duration GRBs for which the simplest version of the afterglow scenario describes a rather extensive multi-epoch and multifrequency data set. In many cases, more data also means a need for a more complicated afterglow scenario. This is independent of the publication bias that afterglows with exciting irregular behavior, such as GRBs 071031 (Krühler et al. 2009), 080129 (Greiner et al. 2009), 081029 (Nardini et al. 2011), 100621A (Greiner et al. 2013), 100814A (Nardini et al. 2014), 111209A (Greiner et al. 2015a; Kann et al. 2018), get more easily published than standard GRB afterglows. It remains to be investigated whether some of the standard afterglows can be fitted with the next-simplest version of afterglow models. The hydrodynamical simulations including the incorporation of the off-axis angle view (van Eerten 2015) are one way, and analytical jet spreading models are another.

While there is a wealth of published papers dealing with the fireball modeling of individual GRB afterglows, the vast majority only allow consistency checks, since the data are not sufficient to derive all five model parameters (plus redshift). The three historical exceptions for which all parameters could be determined are GRBs 980703 (Frail et al. 2003), 000926 (Harrison et al. 2001), and 090323 (Cenko et al. 2011). More recently, our group managed to add another four GRBs to this sample: 100418A, 110715A, 130418A (Varela 2017), and 121024A (see Varela et al. 2016 for details). This small sample, out of a total of $>700$ known X-ray/optical afterglows, shows the challenge of testing the afterglow model(s). And even these seven GRB afterglows are not uniquely described by a single set of parameters or the simplest fireball version: one GRB is equally well described by either wind or ISM density profile, two other GRBs show substantial flaring activity implying additional energy injection, and another two GRBs show strong evidence of an inverse Compton component. There are indications in this sample for a preference of a wind-like GRB environment,

http://www . mpe.mpg.de/ jcg/grbgen.html contrary to the results of many early analyses. However, this topic-and other issues related to afterglows like a reliable distribution of microphysical parameters-require a substantially larger sample size.

From an observer's point of view, it is not obvious how best to reach a larger sample size, i.e., what a guaranteed-success strategy would look like. Radio observations are only meaningful at late times when scintillation has ceased, but then the X-ray and optical-NIR instrumentation typically is not sensitive enough to detect the afterglow any longer. However, radio observations provide crucial constraints for the afterglow modeling. Alternatively, dedicated multi-band, multi-epoch ALMA monitoring seems promising for two reasons: First, it is sensitive enough to cover a larger time interval of the afterglow emission (2-3 weeks). Second, the $v_{m}$-crossing is faster than that of $v_{c}$, allowing (in combination with the decay slope) a potentially better distinction between wind and ISM environment. While rapid (within a day) target-of-opportunity (ToO) observations are allowed with ALMA, the general acceptance level of GRB-related ToO proposals is going down after more than a decade of Swift-driven afterglow studies, and the need for proposals to be accepted at several observatories during the same semester does not make things easier (see, e.g., Middleton et al. 2017 for a description of the problem and suggested solutions). Instead of attempting full multiwavelength coverage over a long time interval, a graded approach with dense X-ray/optical/sub-mm coverage during the first days and sub-mm/radio at later stages might be a better approach. This is particularly motivated by the potential of trans-relativistic dynamical models and models including jet dynamics that improve upon earlier closure relations. The number of open questions and the impact that a proper knowledge of the GRB afterglow emission process would have on a variety of other astrophysical areas certainly justifies a concerted approach.

Acknowledgements. During the writing of this paper, the radio astronomy community lost a great scientist, and we lost a dear colleague and collaborator, Ger de Bruyn. His insights in radio scintillation were crucial in the work presented in this paper, and he will be missed by many. J. G. is particularly grateful to Phil Edwards for scheduling the many ATCA ToO observations. S. K., A. N. G., S. S., and D. A. K. acknowledge support by DFG grant Kl 766/16-1. D. A. K. acknowledges financial support from the Spanish research project AYA 201458381-P, and from Juan de la Cierva Incorporación fellowships IJCI-2015-26153 and IJCI-2014-21669. J. F. G., T. K., and P. W. acknowledge support through the Sofja Kovalevskaja award to P. Schady from the A. von Humboldt foundation of Germany. Part of the funding for GROND (both hardware and personnel) was generously granted from the Leibniz-Prize to Prof. G. Hasinger (DFG grant HA 1850/28-1). The Australia Telescope Compact Array is part of the Australia Telescope National Facility, which is funded by the Commonwealth of Australia for operation as a National Facility managed by CSIRO. ALMA is a partnership of ESO (representing its member states), NSF (USA), and NINS (Japan), together with NRC (Canada), NSC and ASIAA (Taiwan), and KASI (Republic of Korea), in cooperation with the Republic of Chile. The Joint ALMA Observatory is operated by ESO, AUI/NRAO, and NAOJ. This work made use of data supplied by the UK Swift Science Data Centre at the University of Leicester. Facilities: Max Planck:2.2 m (GROND), ATCA, ALMA, Swift

\section{References}

Barthelmy, S. D., Barbier, L. M., Cummings, J. R., et al. 2005, Space Sci. Rev. 120, 143

Bignall, H. E., Macquart, J.-P., Jauncey, D. L., et al. 2006, ApJ, 652, 1050

Bolmer, J., Greiner, J., Krühler, T., et al. 2018, A\&A, 609, A62

Breeveld, A. A., Ukwatta, T. N., 2015, GCN Circ. \#18517

Buckley, D., Potter, S., Kniazev, A., et al. 2015, GCN Circ. \#18511

Burrows, D. N., Hill, J. E., Nousek, J. A. et al. 2005, Space Sci. Rev., 120, 165

Cenko, S. B., Frail, D. A., Harrison, F. A., et al. 2010, ApJ, 711, 641

Cenko, S. B., Frail, D. A., Harrison, F. A., et al. 2011, ApJ, 732, 29

Chandra, P., \& Frail, D. A. 2012, ApJ, 746, 156

Chandra, P., Cenko, S. B., Frail, D. A., et al. 2008, ApJ, 683, 924 
Cordes, J. M., \& Lazio, T. J. W. 2002 ArXiv e-prints [arXiv:astro-ph/ Q207156]

de Bruyn, A. G., \& Macquart, J.-P. 2015, A\&A, 574, A125

Dainotti, M. G., Hernandez, X., Postnov, S., et al. 2017, ApJ, 848, 88

Dennett-Thorpe, J., \& de Bruyn, A. G. 2002, Nature, 415, 57

Dichiara, S., Kopac, D., Guidorzi, C., Kobayashi, S., Gomboc, A., 2015, GCN Circ. \#18520

Frail, D. A., Kulkarni, S. R., Nicastro, L., Feroci, M., Taylor, G. B., 1997, Nature, 389,261

Frail, D. A., Waxman, E., \& Kulkarni, S. R. 2000, ApJ, 537, 191

Frail, D. A., Yost, S. A., Berger, E. et al. 2003, ApJ, 590, 992

Gehrels, N., Chincarini, G., Giommi, P., et al. 2004, ApJ, 621, 558

Goodman, J. 1997, New Astron., 2, 449

Granot, J., \& Sari, R. 2002, ApJ, 568, 820

Granot, J., \& van der Horst, A. J. 2014, PASA, 31, e008

Granot, J., Königl, A., Piran, T. 2006, MNRAS, 370, 1946

Greiner, J., Bornemann, W., Clemens, C., et al. 2008, PASP, 120, 405

Greiner, J., Krühler, T., McBreen, S., et al. 2009, ApJ, 693, 1912

Greiner, J., Krühler, T., Nardini, M., et al. 2013, A\&A 560, A70

Greiner, J., Mazzali, P. A., Kann, D. A., et al. 2015, Nature, 523, 189

Greiner, J., Wieringa, M., Wiseman, P. 2015b, GCN Circ. \#18548

Harrison, F. A., Yost, S. A., Sari, R. et al. 2001, ApJ, 559, 123

Hjorth, J., Sollermann, J., Moller, P. et al. 2003, Nature, 423, 847

Kann, D. A., Schady, P., Olivares, E. F. et al. 2018, A\&A, submitted [arXiv:1706.00601]

Krühler, T., Kücü Yoldaş, A., Greiner, J., et al. 2008, ApJ, 685, 376

Krühler, T., Greiner, J., McBreen, S., et al. 2009, ApJ, 697, 758

Küpcü Yoldaş, A., Krühler, T., Greiner, J., et al. 2008, AIP Conf. Proc., 1000, 227

Laskar, T., Berger, E., Tanvir, N. R., et al. 2014, ApJ, 781, 1

Malesani, D., Tanvir, N. R., Xu, D., et al. 2015, GCN Circ. \#18501

Macquart, J.-P., \& de Bruyn, A. G. 2007, MNRAS, 380, L20

McBreen, S., Krühler, T., Rau, A. et al. 2010, A\&A 516, A71
McMullin, J. P., Waters, B., Schiebel, D., Young, W., Golap, K., 2007, Astron. Data Analysis Software and Systems XVI, eds. R. A. Shaw, F. Hill, \& D. J. Bell ASP Conf. Ser., 376, 127

Meszaros, P., \& Rees, M. J., 1997, ApJ, 476, 232

Middleton, M. J., Casella, P., Ghandi, P. et al. 2017, New Astron. Rev. 79, 26

Nardini, M., Greiner, J., Krühler, T. et al. 2011, A\&A, 531, A39

Nardini, M., Elliott, J., Filgas, R., et al. 2014, A\&A, 562, A29

Osborne, J. P., Beardmore, A. P., Evans, P. A., Goad, M. R. 2015, GCN Circ. \#18504

Panaitescu, A., \& Kumar, P. 2002, ApJ, 571, 779

Petry, D., CASA Development Team 2012, Astron. Data Analysis Software and Systems XXI. Proc. of Conf. Paris, 2011, eds. P. Ballester, D. Egret, N. P. F. Lorente, ASP Conf. Ser., 461, 849

Rickett, B. J. 1990, ARA\&A, 28, 561

Sakamoto, T., Barthelmy, S. D., Cummings, J. R., et al. 2015, GCN Circ. \#18514

Sault, R. J., Teuben, P. J., Wright, M. C. H. 1995, in Astronomical Data Analysis Software and Systems IV, eds. R. Shaw, et al., ASP Conf. Ser., 77, 433

Schlafly, E. F., \& Finkbeiner, D. P. 2011, ApJ, 737, 103

Stanek, K. Z., Matheson, T., Garnavich, P. M. et al. 2003, ApJ, 591, L17

Taylor, G. B., Frail, D. A., Beasley, A. J., Kulkarni, S. R. 1997, Nature, 389, 263

Tody, D. 1993, in Astronomical Data Analysis Software and Systems II, eds. R. J. Hanisch, R. J. V. Brissenden, \& J. Barnes, ASP Conf. Ser., 52, 173

Ukwatta, T. N., Barthelmy, S. D., Baumgartner, W. H. 2015, GCN Circ. \#18499

van der Horst, A. J., Paragi, Z., de Bruyn, A. G., et al. 2014, MNRAS, 444, 3151 van Eerten, H. 2015, J. High-Energy Astrophys., 7, 23

Varela, K., 2017, Dissertation, TU Munich, Germany

Varela, K., van Eerten, H., Greiner, J. et al. 2016, A\&A, 589, A37

Walker, M. A. 1998, MNRAS, 294, 307

Watson, A. M., Butler, N., Kutyrev, A., et al. 2015, GCN Circ. \#18512

Xu, D., Tanvir, N. R., Malesani, D., Fynbo, J. P. U., et al. 2015, GCN Circ. \#18505

Yoon, S.-C., Dierks, A., Langer, N., et al. 2012, A\&A, 542, A113

Yost, S. A., Harrison, F. A., Sari, R., Frail, D. A., et al. 2003, ApJ, 597, 459

\section{Appendix A: Secondary standard stars}

Table A.1. Secondary standard stars used to calibrate the optical-NIR afterglow measurements.

\begin{tabular}{ccccccc}
\hline \hline $\begin{array}{c}\text { RA/Dec (deg) } \\
(2000.0)\end{array}$ & $\begin{array}{c}\text { RA/Dec (hms) } \\
(2000.0)\end{array}$ & $\begin{array}{c}g^{\prime} \\
(\mathrm{mag})\end{array}$ & $\begin{array}{c}r^{\prime} \\
(\mathrm{mag})\end{array}$ & $\begin{array}{c}i^{\prime} \\
(\mathrm{mag})\end{array}$ & $\begin{array}{c}z^{\prime} \\
(\mathrm{mag})\end{array}$ & $\begin{array}{c}J \\
(\mathrm{mag})\end{array}$ \\
$(\mathrm{mag})$ & $\begin{array}{c}K_{\mathrm{s}} \\
(\mathrm{mag})\end{array}$ \\
\hline
\end{tabular}

$76.22154-6.41494 \quad 05: 04: 53.17-06: 24: 53.8$ $76.23341-6.42239 \quad 05: 04: 56.02-06: 25: 20.6$ $76.18746-6.43186 \quad 05: 04: 44.99-06: 25: 54.7$ $76.18821-6.43417 \quad 05: 04: 45.17-06: 26: 03.0$ $76.22938-6.43628 \quad 05: 04: 55.05-06: 26: 10.6$ $76.20600-6.44189 \quad 05: 04: 49.44-06: 26: 30.8$ $76.20441-6.44311 \quad 05: 04: 49.06-06: 26: 35.2$ $76.24538-6.44383 \quad 05: 04: 58.89-06: 26: 37.8$ $76.24888-6.44475 \quad 05: 04: 59.73-06: 26: 41.1$ $76.24046-6.44611 \quad 05: 04: 57.71-06: 26: 46.0$ $76.24809-6.44664 \quad 05: 04: 59.54-06: 26: 47.9$ $76.24358-6.45283 \quad 05: 04: 58.46-06: 27: 10.2$ $76.20421-6.45503 \quad 05: 04: 49.01-06: 27: 18.1$ $76.24854-6.45628 \quad 05: 04: 59.65-06: 27: 22.6$ $76.24492-6.45639 \quad 05: 04: 58.78-06: 27: 23.0$ $76.20492-6.45661 \quad 05: 04: 49.18-06: 27: 23.8$ $76.23579-6.46542 \quad 05: 04: 56.59-06: 27: 55.5$ $76.20871-6.46989 \quad 05: 04: 50.09-06: 28: 11.6$ $76.22787-6.47075 \quad 05: 04: 54.69-06: 28: 14.7$ $76.25004-6.47411 \quad 05: 05: 00.01-06: 28: 26.8$ $76.21046-6.48039 \quad 05: 04: 50.51-06: 28: 49.4$ $76.20200-6.48617 \quad 05: 04: 48.48-06: 29: 10.2$ $\begin{array}{lllllll}16.838 \pm 0.001 & 15.947 \pm 0.001 & 15.664 \pm 0.001 & 15.440 \pm 0.001 & 14.437 \pm 0.002 & 14.000 \pm 0.002 & 13.860 \pm 0.009\end{array}$ $\begin{array}{lllllll}15.289 \pm 0.001 & 14.961 \pm 0.001 & 14.475 \pm 0.001 & 14.325 \pm 0.001 & 13.375 \pm 0.001 & 13.022 \pm 0.002 & 12.860 \pm 0.004\end{array}$ $\begin{array}{lllllll}15.155 \pm 0.001 & 14.914 \pm 0.001 & 14.441 \pm 0.001 & 14.308 \pm 0.001 & 13.374 \pm 0.001 & 13.023 \pm 0.002 & 13.047 \pm 0.004\end{array}$ $\begin{array}{lllllll}18.943 \pm 0.009 & 18.278 \pm 0.004 & 18.046 \pm 0.005 & 17.858 \pm 0.005 & 16.910 \pm 0.020 & 16.439 \pm 0.027 & 16.338 \pm 0.089\end{array}$ $\begin{array}{lllllll}16.412 \pm 0.001 & 15.793 \pm 0.001 & 15.512 \pm 0.001 & 15.323 \pm 0.001 & 14.381 \pm 0.002 & 13.936 \pm 0.002 & 13.853 \pm 0.009\end{array}$ $\begin{array}{lllllll}19.557 \pm 0.014 & 17.987 \pm 0.003 & 17.294 \pm 0.003 & 16.894 \pm 0.002 & 15.656 \pm 0.005 & 14.906 \pm 0.007 & 14.848 \pm 0.023\end{array}$ $\begin{array}{lllllll}18.429 \pm 0.005 & 16.989 \pm 0.001 & 16.419 \pm 0.001 & 16.063 \pm 0.001 & 14.871 \pm 0.004 & 14.159 \pm 0.004 & 14.115 \pm 0.012\end{array}$ $\begin{array}{lllllll}18.927 \pm 0.007 & 17.926 \pm 0.003 & 17.539 \pm 0.003 & 17.282 \pm 0.003 & 16.177 \pm 0.007 & 15.552 \pm 0.009 & 15.483 \pm 0.037\end{array}$ $\begin{array}{lllllll}18.823 \pm 0.007 & 17.953 \pm 0.003 & 17.592 \pm 0.003 & 17.350 \pm 0.003 & 16.287 \pm 0.009 & 15.775 \pm 0.012 & 15.698 \pm 0.046\end{array}$ $\begin{array}{lllllll}18.042 \pm 0.003 & 17.457 \pm 0.002 & 17.242 \pm 0.002 & 17.068 \pm 0.002 & 16.179 \pm 0.007 & 15.785 \pm 0.012 & 15.733 \pm 0.046\end{array}$ $\begin{array}{lllllll}18.253 \pm 0.004 & 17.550 \pm 0.002 & 17.278 \pm 0.002 & 17.084 \pm 0.003 & 16.097 \pm 0.007 & 15.634 \pm 0.011 & 15.586 \pm 0.039\end{array}$ $\begin{array}{lllllll}16.975 \pm 0.001 & 16.058 \pm 0.001 & 15.684 \pm 0.001 & 15.422 \pm 0.001 & 14.353 \pm 0.002 & 13.896 \pm 0.002 & 13.711 \pm 0.007\end{array}$ $\begin{array}{lllllll}16.637 \pm 0.001 & 16.080 \pm 0.001 & 15.909 \pm 0.001 & 15.760 \pm 0.001 & 14.888 \pm 0.002 & 14.595 \pm 0.005 & 14.589 \pm 0.014\end{array}$ $\begin{array}{lllllll}17.206 \pm 0.002 & 16.637 \pm 0.001 & 16.419 \pm 0.001 & 16.259 \pm 0.001 & 15.334 \pm 0.004 & 15.057 \pm 0.007 & 14.938 \pm 0.018\end{array}$ $\begin{array}{lllllll}19.816 \pm 0.017 & 18.618 \pm 0.005 & 18.125 \pm 0.005 & 17.828 \pm 0.005 & 16.660 \pm 0.014 & 16.090 \pm 0.018\end{array}$ $\begin{array}{lllllll}17.422 \pm 0.002 & 16.985 \pm 0.001 & 16.840 \pm 0.002 & 16.710 \pm 0.002 & 15.832 \pm 0.007 & 15.538 \pm 0.011 & 15.668 \pm 0.039\end{array}$ $\begin{array}{lllllll}17.697 \pm 0.002 & 16.485 \pm 0.001 & 16.035 \pm 0.001 & 15.720 \pm 0.001 & 14.555 \pm 0.002 & 13.993 \pm 0.002 & 13.858 \pm 0.007\end{array}$ $\begin{array}{lllllll}17.155 \pm 0.002 & 15.646 \pm 0.001 & 14.865 \pm 0.001 & 14.477 \pm 0.001 & 13.231 \pm 0.001 & 12.624 \pm 0.001 & 12.455 \pm 0.002\end{array}$ $\begin{array}{lllllll}19.456 \pm 0.014 & 17.722 \pm 0.002 & 16.879 \pm 0.002 & 16.392 \pm 0.001 & 15.037 \pm 0.004 & 14.370 \pm 0.004 & 14.142 \pm 0.009\end{array}$ $\begin{array}{lllllll}14.953 \pm 0.001 & 14.683 \pm 0.001 & 14.181 \pm 0.001 & 13.959 \pm 0.001 & 12.881 \pm 0.001 & 12.503 \pm 0.001 & 12.362 \pm 0.002\end{array}$

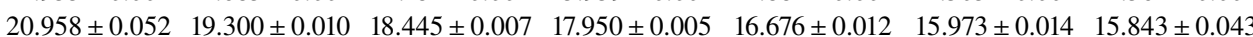

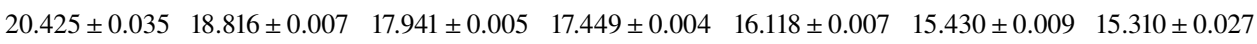

Notes. The $g^{\prime} r^{\prime} i^{\prime} z^{\prime}$ magnitudes are in the AB system, $J H K_{\mathrm{s}}$ in Vega. 